\title{
Evolução histórica do Fator de Impacto (FI) na base Web of Science (WoS) dos periódicos do Brasil entre 2008 e 2018
}

\author{
Impact Factor's historical evolution in the Web of Science (WoS) database of the \\ Brazilian journals between 2008 and 2018
}

\author{
Renata Seabra Domingues \\ Bacharel administração \\ Associação Brasileira de Editores Científicos \\ reseabra@hotmail.com \\ Lucilene Delazari dos Santos \\ Doutora em Biologia Molecular \\ Universidade Estadual Paulista \\ lucilenebio@gmail.com \\ Juliana Siani Simionato \\ Mestre em Teoria e Pesquisa em Comunicação \\ Universidade Estadual Paulista \\ juliana@cevap.unesp.br
Rui Seabra Ferreira Junior
Doutor em doenças tropicais
Universidade Estadual Paulista rui.seabra@unesp.br

\author{
Claudia Vilalva Cassaro \\ Mestre em Doenças Tropicais \\ Universidade Estadual Paulista \\ claudia.v.cassaro@gmail.com
}

\author{
Hélio Rubens de Carvalho Nunes \\ Doutor em Saúde Coletiva \\ Universidade Estadual Paulista \\ brcn@outlook.com.br \\ Milton Shintaku \\ Doutor em Ciência da Informação \\ Instituto Brasileiro de Informação em Ciência e \\ Tecnologia \\ shintaku@ibcit.br
}

\author{
Benedito Barraviera \\ Doutor em Medicina Interna \\ Universidade Estadual Paulista \\ bbviera@gnosis.com.br
}

\begin{abstract}
Resumo Os resultados animadores inserem e globalizam definitivamente a ciência brasileira.

Palavras chave

Comunicação científica. Fator de impacto. Periódicos científicos. Web of Science.
\end{abstract}

O Fator de Impacto, embora não seja unanimidade, é um dos indicadores de qualidade para as revistas indexadas na $W e b$ of Science. Este estudo objetivou apresentar a série histórica do Fator de Impacto das revistas brasileiras indexadas nesta base a partir de 1997. Nesse ano havia 11 periódicos indexados; em 2008, 31; e em 2018, 158. Atualmente 99 revistas tem Fator de Impacto maior que 0.5 (62.7\%); 54 tem Fator de Impacto maior que 1.0 (34.2\%); 10 tem Fator Impacto maior que 2.0 (6.3\%); e duas tem Fator Impacto maior que $3.0(1,3 \%)$. O evidente crescimento desta métrica se deve principalmente a quatro fatores: a crescente exigência do sistema Qualis da CAPES aos programas de Pós-graduação para que priorizem sempre revistas de elevado Fator de Impacto para publicação; o Programa Editorial do CNPq financiando as revistas há quase trinta anos; a consolidação da biblioteca SciELO exigindo padronização e qualidade; e a Associação Brasileira dos Editores Científicos (ABEC) qualificando desde 1985 os Editores brasileiros.

doi: $10.28998 /$ cirev.2020.7ne.01-09

Esta obra está licenciada sob uma Licença Creative Commons.

Submetido em: 27/12/2019

Aceito em: $11 / 03 / 2020$

Publicado em: 30/03/2020 
Renata Seabra Domingues / Claudia Vilalva Cassaro / Lucilene Delazari dos Santos / Hélio Rubens de Carvalho Nunes / Juliana Siani Simionato / Milton Shintaku / Rui Seabra Ferreira Junior /

Benedito Barraviera

\begin{abstract}
The Impact Factor (IF), published since 1972 by the Journal Citation Reports, although not unanimously among researchers, is one of the quality indicators for journals indexed in the Web of Science database. This study aimed to present the IF historical series of Brazilian journals indexed on this base from 1997. At that time there were 11 indexed journals; in 2008, 31; and 2018, 158. Currently 99 journals have IF greater than 0.5 (62.7\%); 54 have IF greater than 1.0 (34.2\%); 10 have IF greater than 2.0 (6.3\%); and both have greater than 3.0 (1.3\%). The evident growth of this metric, especially in the last 10 years, is mainly due to four factors: the growing requirement of CAPES Qualis system for graduate programs to always prioritize indexed, arbitrated and high IF journals for publication of academic research results; CNPq's Editorial Program through public calls for financial support to journals for nearly thirty years; the consolidation of the SciELO library requiring standardization and quality for indexing at its base; and the Brazilian Association of Scientific Editors (ABEC) tirelessly qualifying Brazilian Publishers since 1985 with a view to improving the metrics, indexing and quality of publications. The encouraging results definitively insert and globalize the science published in Brazilian journals in the competitive publishing market, and place Brazil as the largest publishing complex of academic, non-commercial and open access journals in the world.
\end{abstract}

\title{
Keywords:
}

Scientific communication. Impact factor. Scientific journals. Web of science.

\section{INTRODUÇÃO}

As revistas científicas têm mais de 350 anos de existência. Sua evolução transformou esses periódicos em um dos principais canais de disseminação mundial dos resultados de pesquisa. Isto gerou a necessidade de desenvolver uma metodologia capaz de avaliar o impacto e a qualidade das publicações. Gross e Gross, em 1927, sugeriram pela primeira vez contar as citações dos artigos, de forma a classificar a importância das revistas. Tais iniciativas tornaram-se precursoras das métricas para avaliação de periódicos.

Eugene Garfield foi o primeiro a desenvolver uma equação que media o Fator de Impacto (FI), embora somente em 1955 foi desenvolvida uma metodologia que possibilitava o seu cálculo. Em 1961, o FI foi publicado sob a denominação de Science Citation Index (SCI), reordenando o índice de citação do autor no índice de citação do periódico (GARFIELD, 1999). A partir de 1972 a nova métrica passou a ser calculada para as revistas indexadas na base Web of Science (WoS) do Institute for Scientific Information (ISI) e, posteriormente, passou a ser publicada anualmente pelo Journal Citation Reports (JCR).

Desde então o FI tem se tornado um dos principais índices para verificação da qualidade e do impacto das revistas no mundo, incluindo o Brasil. Targino e Garcia (2000) realizaram um amplo estudo sobre a participação nacional na base do ISI, revelando a pequena participação do nosso país. Esse ponto evidencia a necessidade de estudos constantes para verificação da participação brasileira em bases e em relatórios de verificação de impacto, a fim de avaliar mundialmente a evolução dos periódicos brasileiros.

Ainda no âmbito nacional, o FI é um dos principais critérios para a estratificação do Qualis, indicador desenvolvido pela Coordenação de Aperfeiçoamento Pessoal de Nível Superior (CAPES). Foi introduzido em 1998 como instrumento de avaliação da qualidade da produção intelectual dos programas de pós-graduação brasileiros (CAPES, 2008; ANDRADE, GALEMBECK, 2009; CAPES, 2016).

A partir de 2010, o FI passou a ser um dos principais critérios de avaliação (ANDRIOLO et al., 2010; BARATA, 2015). Atualmente o cálculo do Qualis referência é realizado utilizando-se indicadores bibliométricos e seus percentis, entre eles o FI da WoS, o CiteScore da Scopus e o Google Scholar (CAPES, 2008)

O objetivo do presente estudo foi verificar a evolução do FI na base WoS da Clarivate Analytics desde 1997 até 2018, com ênfase especial entre 2008 e 2018. 


\section{METODOLOGIA}

O presente estudo tem abordagem predominantemente quantitativa, visto que trata de indicadores bibliométricos. De acordo com Lopes et al. (2012), a bibliometria é uma técnica quantitativa e estatística para medir índices de produção e disseminação do conhecimento. Nesse sentido, destaca-se neste estudo a verificação do fator de impacto, que é um indicador quantitativo, produzido por medição numérica de citações.

Da mesma forma, possui também aspectos cientométricos, visto que busca verificar a evolução da ciência brasileira em relatórios de impacto. Segundo a definição de Silva e Bianchi (2001), a cientometria é “o estudo da mensuração e quantificação do progresso científico, estando a pesquisa baseada em indicadores bibliométricos". Portanto, este estudo caracteriza-se como cientométrico por utilizar indicadores quantitativos bibliométricos para verificar a evolução dos periódicos brasileiros.

A técnica utilizada para a coleta de dados foi a pesquisa documental, tendo como fonte principal os relatórios publicados anualmente pelo JCR. Assim, acessaram-se os relatórios, selecionando-se os dados de todas as revistas brasileiras a fim de se verificar quais delas estão presentes na base do WoS desde 1997.

Definiram-se como revistas consolidadas aquelas que nunca perderam o FI anualmente publicado. Em consolidação são aquelas que entraram na Base WoS a partir de 2008 e também não perderam a divulgação desta métrica até o momento. A Figura 1 demonstra o percurso da pesquisa para se chegar no corpus de revistas analisadas.

Figura 1. Fluxograma das revistas analisadas entre 2008 e 2018.

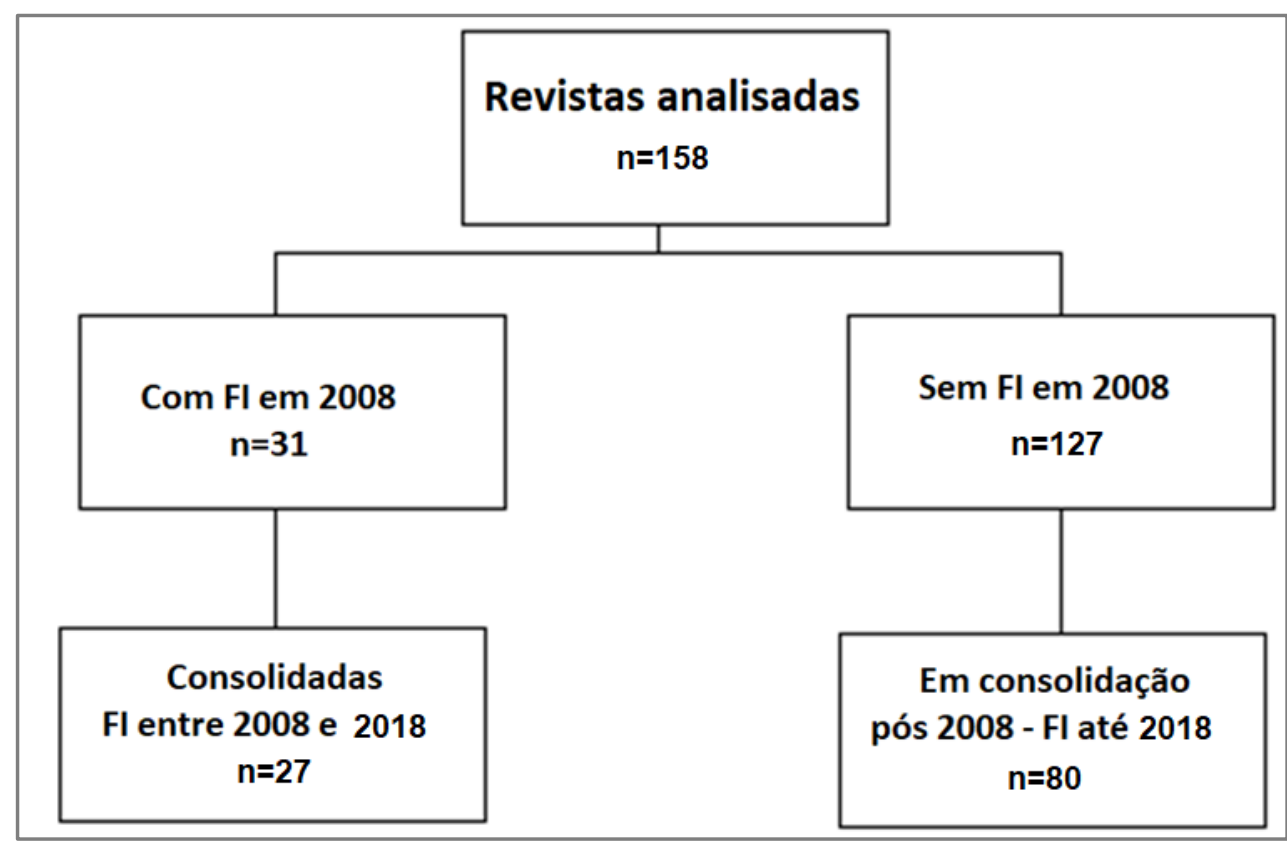

Fonte: elaborado pelos autores (2019)

\section{RESULTADOS E DISCUSSÃO}

Em 1997 estavam indexadas na base WoS 11 periódicos brasileiros, a saber: Memórias do Instituto Oswaldo Cruz, Journal of Brazilian Chemical Society, Brazilian Journal of Medical and Biological Research, Revista de Saúde Pública, Pesquisa Agropecuária Brasileira, Dados - revista de ciências 
sociais, Computational and Applied Mathematics, Brazilian Journal of Genetics, Revista Brasileira de Genética, Arquivos de Biologia e Tecnologia, e Revista de Microbiologia. A média do FI no ano seguinte ao ingresso na base (1998) foi de 0.21, variando entre 0.05 e 0.46 .

Em 2008 havia 31 revistas indexadas na base WoS. Destas, 27 foram consideradas consolidadas, ou seja, estavam na base em 2008 e continuaram até 2018 sem interrupção na publicação anual do FI. São elas: Anais da Academia Brasileira de Ciências, Arquivo Brasileiro de Medicina Veterinária e Zootecnia, Brazilian Archives of Biology and Technology, Brazilian Journal of Chemical Engineering, Brazilian Journal of Medical and Biological Research, Brazilian Journal of Microbiology, Brazilian Journal of Physics, Bulletin of the Brazilian Mathematical Society, Ciência Florestal, Dados Revista de Ciências Sociais, Genetics and Molecular Biology, Iheringia Serie Zoologia, Journal of the Brazillian Chemical Society, Journal of Venomous Animals and Toxins including Tropical Diseases, Memórias do Instituto Oswaldo Cruz, Neotropical Entomology, Neotropical Ichthyology, Química Nova, Pesquisa Agropecuária Brasileira, Revista Brasileira de Ciência do Solo, Revista Brasileira de Entomologia, Revista Brasileira de Psiquiatria, Revista de Saúde Pública, Revista da Sociedade Brasileira de Medicina Tropical e Scientia Agricola.

Em 2018 havia 158 revistas indexadas, sendo que 127 não tinham FI em 2008. Oitenta delas, denominadas "em consolidação", adquiriram o FI durante estes últimos 10 anos e continuam com o mesmo sem interrupção até o momento. Em 2019 o InCites JCR publicou o FI de 2018 de todas as revistas indexadas na referida base. A Tabela 1 e a Figura 2 descrevem em detalhes a evolução do FI das 158 revistas indexadas atualmente na base WoS.

Tabela 1. Evolução do FI das revistas brasileiras entre 2008 e 2018

\begin{tabular}{ccccccccc}
\hline & \multicolumn{2}{c}{ Revista com } & \multicolumn{2}{c}{ Revista com FI } & \multicolumn{2}{c}{ Revista com FI } & \multicolumn{2}{c}{ Revista com FI } \\
Ano & $\mathrm{n}$ & $\%$ & $\mathrm{n}$ & $\%$ & $\mathrm{n}$ & $\%$ & $\mathrm{n}$ & $\%$ \\
\hline 2008 & 17 & 10.8 & 4 & 2.5 & 0 & 0.0 & 0 & 0.0 \\
2009 & 32 & 20.3 & 10 & 6.3 & 1 & 0.6 & 0 & 0.0 \\
2010 & 48 & 30.4 & 12 & 7.6 & 1 & 0.6 & 0 & 0.0 \\
2011 & 52 & 32.9 & 14 & 8.9 & 2 & 1.3 & 0 & 0.0 \\
2012 & 57 & 36.1 & 12 & 7.6 & 0 & 0.0 & 0 & 0.0 \\
2013 & 61 & 38.6 & 13 & 8.2 & 1 & 0.6 & 0 & 0.0 \\
2014 & 68 & 43.0 & 16 & 10.1 & 1 & 0.6 & 0 & 0.0 \\
2015 & 69 & 43.7 & 18 & 11.4 & 3 & 1.9 & 0 & 0.0 \\
2016 & 71 & 44.9 & 32 & 20.3 & 5 & 3.2 & 0 & 0.0 \\
2017 & 87 & 55.1 & 38 & 24.1 & 6 & 3.8 & 1 & 0.6 \\
2018 & 99 & 62.7 & 54 & 34.2 & 10 & 6.3 & 2 & 1.3 \\
\hline
\end{tabular}

Fonte: dados da pesquisa (2019) 
Figura 2. Evolução do FI dos periódicos brasileiros entre 2008 e 2018

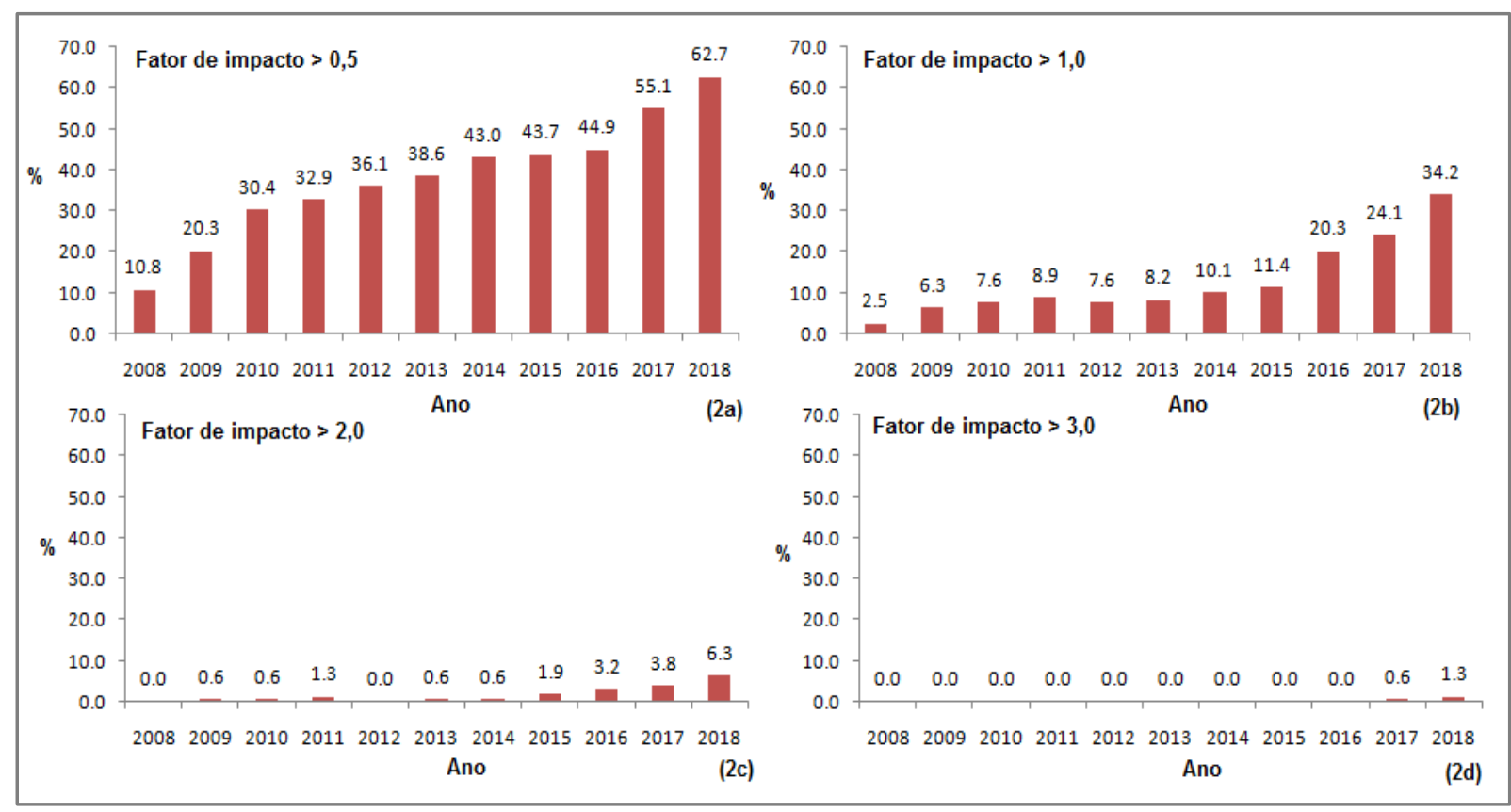

Fonte: dados da pesquisa (2019)

Atualmente, das 158 revistas brasileiras indexadas, 99 tem FI $>0.5$ (62.7\%), 54 com FI $>1.0$ (34.2\%), 10 com FI>2.0 (6.3\%) e 2 com FI>3.0, que corresponde a 1.3\%.

As duas revistas brasileiras com FI acima de 3.0 são publicadas com apoio da Elsevier. A revista Natureza \& Conservação, também denominada de Braz̧ilian Journal of Nature Conservation, alcançou em 2018 o FI de 5.176, embora seja de acesso restrito segundo a plataforma do JCR. O seu editor-chefe é professor titular do Departamento de Ecologia da Universidade de São Paulo. A segunda revista brasileira, denominada Journal of Materials Research and Technology (JMR\&T), é a única revista brasileira de acesso aberto com FI acima de 3.0. Em 2018 alcançou o FI de 3.327. É vinculada à Associação Brasileira de Metalurgia, Materiais e Mineração e coordenada por um editorchefe cedido pela University of California (USA). Como é sabido, participar do portfólio de um publisher internacional sempre dá prestígio e visibilidade, embora os investimentos sejam elevados para manutenção da publicação.

Cabe destacar alguns pontos importantes revelados no presente estudo. Primeiramente, grande parte das revistas com FI ainda estão em estrato inicial, ou seja, com FI > 0.5. Em 2018, os resultados apontaram que 99 revistas $(62.7 \%)$ estão no primeiro estrato, ou seja, com FI em ascensão. Mesmo com o aumento da visibilidade internacional apresentado nesses 10 anos, o índice de citação ainda é baixo, se considerarmos países com maior presença como os Estados Unidos, Inglaterra e Alemanha, por exemplo.

Independentemente do estrato em que se posicionam, ressalta-se neste estudo o crescimento da presença das revistas nacionais em indexadores internacionais como o WoS, possibilitando o incremento do seu FI. Mesmo que haja muitas críticas em relação ao FI e ao WoS, ter 158 revistas nacionais no JCR é um indicador substancial de melhoria da qualidade das publicações brasileiras.

O crescimento considerável do FI nos últimos dez anos pode ser atribuído a quatro fatores principais, a saber: 
(1) A criação da ABEC. A Associação Brasileira dos Editores Científicos (ABEC) foi criada em 1985 por um grupo de Editores visionários. Assim, vem qualificando incansavelmente os Editores brasileiros com vistas à melhoria das métricas, das indexações e da qualidade das publicações científicas desde então. A ABEC ministrou, ao longo destes últimos 34 anos de existência, 26 cursos de capacitação em editoração científica, contribuindo de forma ímpar para o crescimento da qualidade e da citação da ciência brasileira em âmbito internacional.

(2) A implantação do sistema Qualis da CAPES. De acordo com BARATA (2016), o sistema de avaliação dos programas de pós-graduação foi instituído no Brasil pela Coordenação de Aperfeiçoamento de Pessoal do Ensino Superior (CAPES) em 1977. Naquela época a avaliação era expressa em conceitos: A (muito bom), B (bom), C (regular), D (fraco) e E (insuficiente) (FERREIRA; MOREIRA, 2002). Em 1990, os conceitos foram substituídos por notas de 1 a 5, e passaram a ser incluídos no processo de avaliação alguns indicadores quantitativos, entre os quais a quantidade de artigos publicados pelos programas (FERREIRA; MOREIRA, 2002). Em 1998, ocorreu mudança substancial no processo, com a padronização da ficha de avaliação, que incluía sete quesitos, a saber: a proposta do programa, o corpo docente, as atividades de pesquisa, as atividades de formação, o corpo discente, as teses e dissertações e a produção intelectual. Todas as áreas de avaliação deveriam analisar os mesmos quesitos, embora pudessem utilizar, no processo, diferentes tipos de indicadores (BARATA, 2015). Naquela oportunidade a classificação adotada dividia os periódicos em três grupos com três estratos cada. Os grupos separavam os periódicos segundo a circulação - internacional, nacional ou local - e, em cada grupo, as revistas científicas eram classificadas nos estratos $\mathrm{A}, \mathrm{B}$ e C, conforme seu impacto ou relevância para determinada área científica. Durante dez anos, essa classificação foi adotada no processo de avaliação, sofrendo diversos ajustes em cada período avaliado.

Em 2007, a Diretoria de Avaliação propôs a reformulação do Qualis com base em uma avaliação quantitativa que mostrava o uso inadequado da classificação anterior e a perda progressiva do poder discriminatório ao longo dos anos. Muitas áreas acabavam efetivamente utilizando três ou quatro estratos na avaliação, e poucas eram aquelas que usavam os nove estratos previstos (BARATA, 2015). Após praticamente um ano de intensas discussões, foi aprovada a nova classificação contendo sete estratos, a saber: A1, A2, B1, B2, B3, B4 e B5. Havia ainda o estrato C, destinado às publicações que não constituíam periódicos científicos ou não atendiam aos critérios mínimos estabelecidos em cada área. Naquela oportunidade, o FI publicado anualmente pelo JCR da atual Clarivate Analytics passou ser um dos critérios indicativos de qualidade.

Barata (2016) finaliza sua análise concluindo que o sistema Qualis é uma das principais ferramentas utilizadas para se avaliar os programas de pós-graduação no Brasil. Sua função é auxiliar os comitês de avaliação nos processos de análise e de qualificação da produção bibliográfica dos docentes e discentes dos programas credenciados pela Capes. Estas exigências crescentes, em que pese serem criticadas por muitos, acabaram indiretamente contribuindo com o incremento do FI dos periódicos nacionais.

(3) Programa Editorial do CNPq. O objetivo do Programa é apoiar e incentivar a editoração e a publicação de periódicos científicos brasileiros em todas as áreas do conhecimento, sendo considerado prioritário o apoio às revistas divulgadas por meio eletrônico e acesso aberto, ou de forma impressa/eletrônica simultaneamente (http://memoria.cnpq.br/programa-editorial). O Conselho Nacional de Desenvolvimento Científico e Tecnológico $(\mathrm{CNPq})$ vem atuando na área de maneira sistemática, com chamadas anuais, há quase trinta anos. A partir de 2006, ganhou considerável reforço com a parceria da Coordenação de Aperfeiçoamento de Pessoal de Nível Superior (CAPES), 
que passou a participar com 50\% dos recursos financeiros aprovados para o Programa. Hoje, é possível afirmar que o Programa contempla o que há de excelência na área. As publicações submetidas à chamada devem ser mantidas e editadas por instituição, associação ou sociedade científica brasileira sem fins lucrativos e contribuir para elevar o nível de qualidade, forma e conteúdo das revistas científicas nacionais dedicadas à Ciência, Tecnologia e Inovação. O subsídio desta respeitável Instituição de apoio à pesquisa dispensa elogios, haja vista sua contribuição direta para o crescimento do FI dos periódicos brasileiros.

(4) Criação da Biblioteca Digital SciELO. Em 1998 foi criado o projeto Scientific Electronic Library Online (SciELO), uma biblioteca digital de acesso aberto e modelo cooperativo de publicação digital de periódicos científicos brasileiros. É o resultado de um projeto de pesquisa da Fundação de Amparo à Pesquisa do Estado de São Paulo (FAPESP), em parceria com a Centro Latino-Americano e do Caribe de Informação em Ciências da Saúde (BIREME). Desde 2002 conta também com o apoio do CNPq. O Projeto tem por objetivo o desenvolvimento de uma metodologia comum para a preparação, armazenamento, disseminação e avaliação da produção científica em formato digital. (https://pt.wikipedia.org/wiki/Scientific Electronic Library Online)

O Comitê Consultivo SciELO Brasil adota os critérios, a política e os procedimentos para a admissão e a permanência de periódicos científicos na coleção SciELO Brasil (versão de 2004 http://www.scielo.br/criteria/scielo brasil pt.html) e tem por missão:

- O aperfeiçoamento do caráter científico, desempenho, impacto e influência da Coleção como um todo, dos periódicos individualmente e das pesquisas que comunicam:

- A inclusão de novos periódicos na Coleção;

- A exclusão de periódicos da Coleção;

- A avaliação periódica do desempenho da Coleção como um todo e dos periódicos individualmente, propondo recomendações para seu aperfeiçoamento;

- A atualização dos critérios de avaliação para admissão e permanência de periódicos na Coleção; e

- A definição e aperfeiçoamento do funcionamento do Comitê, de modo a cumprir de modo eficiente os objetivos anteriores.

O evidente crescimento do Fator de Impacto das revistas brasileiras, em especial nos últimos 10 anos, se deve principalmente a essas instituições, que direta ou indiretamente passaram a exigir qualidade, competitividade e sustentabilidade dos periódicos brasileiros. Tais resultados animadores inserem e globalizam definitivamente a ciência publicada nas revistas brasileiras no competitivo mercado editorial e colocam o Brasil como o maior complexo editorial de revistas acadêmicas, não comerciais e de acesso aberto do mundo.

\section{CONSIDERAÇÕES FINAIS}

O Brasil possui indubitavelmente o maior complexo Editorial, acadêmico, não comercial e de acesso aberto do mundo. Este estudo apresenta a evolução do FT das revistas brasileiras indexadas na base WoS e publicado pelo JRC desde 1997. Atualmente temos 158 revistas indexadas, sendo 99 com FI acima de 0.5 (62.7\%), 54 com Fator de Impacto maior que 1.0 (34.2\%), 10 com FI maior que $2.0(6.3 \%)$ e duas com FI acima de $3.0(1,3 \%)$.

Consideramos que o evidente crescimento desta métrica, especialmente nos últimos 10 anos, se deve principalmente a quatro fatores: à crescente exigência do sistema Qualis da CAPES aos programas de Pós-graduação para que priorizem sempre revistas indexadas, arbitradas e de elevado FI para publicação dos resultados das pesquisas; ao Programa Editorial do CNPq por meio 
de chamadas públicas para apoio financeiro às revistas há quase trinta anos; à consolidação da biblioteca SciELO, exigindo padronização e qualidade para indexação na sua base; e a ABEC, qualificando desde 1985 os Editores brasileiros com vistas à melhoria das métricas, das indexações e da qualidade das publicações.

Os resultados animadores inserem e globalizam definitivamente a ciência publicada nas revistas brasileiras no competitivo mercado editorial. Salientamos, por fim, a necessária continuidade do apoio editorial aos periódicos brasileiros pelas agências de fomento, em especial à CAPES e ao CNPq a fim de que continuem crescendo nas métricas, tornem-se cada vez mais internacionalmente competitivos e consolidem a sustentabilidade a médio prazo.

\section{REFERÊNCIAS}

ANDRADE, J. B. de; GALEMBECK, F. QUALIS: Quo Vadis?. Quím. Nova, São Paulo, v. 32, n. 1, p. 5, 2009.

ANDRIOLO A. et al. Classification of Journals in the QUALIS system of CAPES - URGENT need of changing the criteria!. J. Venom. Anim. Toxins incl. Trop. Dis, v. 16, n. 3, p. 391-394, 2010.

BARATA, R. B. A Abrasco e a Pós-Graduação Stricto Sensu em Saúde Coletiva. In: LIMA, Nisia Trindade, SANTANA, José Paranaguá, PAIVA, Carlos Henrique Assunção, orgs. Saúde coletiva: a Abrasco em 35 anos de história [online]. Rio de Janeiro: Editora FIOCRUZ, p. 169198, 2015.

BARATA, R. de C. B. Dez coisas que você deveria saber sobre o Qualis. Revista Brasileira de Pós-graduação, [s.l.], v. 13, n. 30, p.13-40, 11 ago. 2016. CAPES.

http://dx.doi.org/10.21713/2358-2332.2016.v13.947.

BRAMBILLA, S. D. S.; STUMPF, I. R. C. Produção científica da UFRGS representada na WOS (2000-2009). Perspectivas em Ciência da Informação, v. 17, n. 3, p. 34-50, 2012.

BRASIL. Ministério da Educação (MEC). Coordenação de Aperfeiçoamento de Pessoal de Nível Superior (Capes). Diretoria de Avaliação, Reestruturação do Qualis. 2008. Disponível em: https://www.capes.gov.br/images/stories/download/avaliacao/Restruturacao Qualis.pdf. Acesso em: 28 ago. 2019.

Ministério da Educação (MEC). Coordenação de Aperfeiçoamento de Pessoal de Nível Superior (CAPES). Considerações sobre Qualis Periódicos. 2016. Disponível em: https://www.capes.gov.br/images/documentos/Qualis periodicos 2016/Direito Qualis .pdf. Acesso em: 28 ago. 2019.

DA SILVA, J. A.; BIANCHI, M. de L. P. Cientometria: a métrica da ciência. Paidéia (Ribeirão Preto), v. 11, n. 21, p. 5-10, 2001.

FERREIRA, M. M.; MOREIRA, R. L (Orgs.). Capes. 50 anos: depoimento ao CPDOC/ FGV. Brasilia: FGV/CPDOC/CAPES, 2002. Disponível em: http://www.dominiopublico.gov.br/ download/texto/me001600.pdf. Acesso em: 27 dez. 2019.

GARFIELD, E. Journal impact factor: a brief review. CMAJ, v. 161. n. 8, p. 979-980, 1999.

GRÁCIO, M. C. C.; OLIVEIRA, E. F. T. de. Indicadores cientométricos normalizados: um estudo na produção científica brasileira internacional (1996 a 2011). Perspectivas em Ciência da Informação, v. 19, n. 3, p. 118-133, 2014. 
GROSS, P. L.K.; GROSS, E. M. College libraries and chemical education. Science, v. 66, n. 1713, p. 385-389, 1927.

LOPES, S. et al. A Bibliometria e a Avaliação da Produção Científica: indicadores e ferramentas. In: Actas do congresso Nacional de bibliotecários, arquivistas e documentalistas. 2012.

TARGINO, M. das G.; GARCIA, J. C. R. Ciência brasileira na base de dados do Institute for Scientific Information (ISI). Ciência da Informação, v. 29, n. 1, p. 103-117, 2000. 